\title{
Influence of the discharge time of solution plasma process on the formation of gold nanoparticles in alginate matrix
}

Anyarat Watthanaphanit ${ }^{\mathrm{a}, \mathrm{c}, *}$, Yong Kang $\mathrm{Heo}^{\mathrm{c}}$, and Nagahiro Saito ${ }^{\mathrm{a}, \mathrm{b}, \mathrm{c}}$

\author{
a Social Innovation Design Center (SIDC) Institute of Innovation for Future Society, Nagoya \\ University, Nagoya 464-8603, Japan \\ ${ }^{\text {b } G r e e n ~ M o b i l i t y ~ C o l l a b o r a t i v e ~ R e s e a r c h ~ C e n t e r, ~ N a g o y a ~ U n i v e r s i t y, ~ N a g o y a ~ 464-8603, ~ J a p a n ~}$ \\ ${ }^{c}$ Department of Materials, Physics and Energy Engineering, Graduate School of Engineering,
} Nagoya University, Nagoya 464-8603, Japan

*Corresponding author. E-mail address: anyarat@rd.numse.nagoya-u.ac.jp Department of Materials, Physics and Energy Engineering, Graduate School of Engineering, Nagoya University, Nagoya 464-8603, Japan. Tel.: +81-52-789-2796; Fax: +81-52-789-2796.

\section{ABSTRACT}

Gold nanoparticles (AuNPs) stabilized in alginate matrix were synthesized by applying the plasma into alginate aqueous solution containing gold (III) chloride trihydrate precursor. Effect of the solution plasma process (SPP) discharge time on the physical properties, including shape and size, and the optical absorption properties of the synthesized AuNPs were investigated by varying the discharge times to be $0,5,10,20$ and 30 min. Results reveal that the increase in the discharge time leads to a size reduction of the AuNPs. Also, the particles tend to be more spherical. Appearance of the obtained AuNPs-alginate suspensions along with the change in their UV-vis absorption spectra intensity after samples were left standing for several time 
intervals suggest that alginate solution itself can act both as a stabilizer and a reducing agent for the AuNPs formation while the application of plasma helps to improve the controllability of shape and size of the synthesized particles. Furthemore, the method is simple, reproducible, and does not need any manipulative skill. Since the reducing agent is not required, the method is suitable for utilizing in biomedical application.

Keywords: gold nanoparticle, sodium alginate, solution plasma process, discharge time

\section{Introduction}

Owing to their important property to interact with light at a specific wavelength that leads to a phenomenon called surface plasmon resonance (SPR), gold nanoparticles (AuNPs) exhibit powerful potential in medical field as chemical and biological sensing [1]. They have also been widely studied for application in targeted delivery [2] as well as cancer diagnostic and therapeutic agents [3]. Numerous approaches become feasible since the particles are small enough to enter almost all area of the body, for example, blood-brain barrier [4] and blood-retinal barrier [5]. Since the particles would aim to be administered into living organisms, an important issue of utilizing AuNPs in biomedical application is their safety to cells. Biocompatibility of the AuNPs could be improved both by chemical technique through surface functionalization $[6,7]$ or physical passivation through dispersion in biocompatible substances $[8,9]$. For the latter case, sodium alginate—a natural block copolymer extracted from seaweed—is an alternative matrix for the dispersion of the AuNPs $[10,11]$. It is one of the most commonly used substances for the encapsulation of biologicals because of its gel formation ability after dissolution in water. This gel can function to stabilize the AuNPs to prevent particle agglomeration and settlement.

One useful tool for the synthesis of metal nanoparticles is the glow discharge in liquid 
phase named "solution plasma process (SPP)" [12]. The key of the synthesis is the generation of reactive species induced by the plasma in a liquid environment that can lead to the reduction of ions to nanoparticles. Synthesis of the AuNPs by the SPP was studied in water [13] as well as other media such as liquid nitrogen and ethanol [14]. Previously, we have proposed a one-step production of colloidal AuNPs-alginate aqueous suspension by the solution plasma sputtering (SPS) process [15]. The process was named "SPS" since the AuNPs were produced from sputtering of the gold electrodes. Briefly, the plasma was applied through a pair of gold electrodes which were immersed in alginate aqueous solution. By using the SPS strategy, we obtained spherical AuNPs having diameter $-3-5 \mathrm{~nm}$ which were dispersed well in the alginate matrix within a reasonable period of time (more than six months). Apart from endowing biocompatibility to the AuNPs, utilization of the alginate polymer was of great benefit to the production of AuNPs-alginate suspension by the application of plasma: it can promote the generation of plasma in liquid environment and provide colloidal stability to the obtained suspensions. In the present contribution, production of the AuNPs is still based on the application of plasma into alginate aqueous solution. However, the gold (III) chloride trihydrate was used as a gold precursor. We aim to compare this production strategy with the former research [15]. As a first step, influence of the plasma discharge time on the appearance, size, and optical absorption properties of the AuNPs were studied.

\section{Experimental section}

\subsection{Chemicals}

Gold (III) chloride trihydrate $\left(\mathrm{HAuCl}_{4} \cdot 3 \mathrm{H}_{2} \mathrm{O}, \geq 49.0 \% \mathrm{Au}\right.$ basis) purchased from Sigma-Aldrich (Buchs, Switzerland) was used as a gold precursor. Sodium alginate was 
purchased from Kanto Chemical Co., Inc. (Tokyo, Japan). Alginate aqueous solutions were prepared using ultra-pure water from Aquarius water distillation apparatus, RFD250NB, Advantec (Tokyo, Japan). Sodium alginate aqueous solution was prepared at a concentration of $0.5 \% \mathrm{w} / \mathrm{v}$ while concentration of $\mathrm{HAuCl}_{4} \cdot 3 \mathrm{H}_{2} \mathrm{O}$ precursor in the solution was $0.2 \mathrm{mM}$.

\subsection{Experimental setup and production procedure}

Experimental setup of the SPP used for the production of AuNPs-alginate aqueous suspensions is schematically shown in Fig. 1. Two tungsten rods were used as electrodes. Each electrode was cover with a ceramic tube which was inserted in a silicone stopper. They were then placed in a $100 \mathrm{~mL}$ glass beaker having an inner diameter of $43 \mathrm{~mm}$ and a height of $75 \mathrm{~mm}$. The distance between the tips of electrodes was set to $0.3 \mathrm{~mm}$. Before the discharge, the glass beaker was filled with $90 \mathrm{~mL}$ alginate aqueous solution containing gold (III) chloride trihydrate $(0.2 \mathrm{mM})$. The plasma was generated, at atmospheric pressure and room temperature, using a bipolar-DC pulsed power supply (SPIK 2000A/KT-IDP-1010S). The pulse width and frequency employed during the generation of plasma were $2 \mu \mathrm{s}$ and $15 \mathrm{kHz}$, respectively. Solutions were stirred continuously during the operation in order to maintain homogeneity at any instant.

\subsection{Characterizations}

During the generation of plasma, optical emission spectra were collected using an optical emission spectroscope (AvaSpec-3648, Avantes, USA), operated with an integration time of $10 \mathrm{~ms}$ and average 10 scans within the wavelength range from 200 to $1000 \mathrm{~nm}$. Optical absorption properties of the AuNPs-alginate aqueous suspensions were analyzed with a UV-3600 spectrophotometer (Shimadzu, Japan) in the spectral range from 200 to $800 \mathrm{~nm}$. Samples were prepared by diluting the suspension to reach a concentration of $0.2 \% \mathrm{w} / \mathrm{v}$ 
(calculated based on the concentration of alginate solutions). Transmission electron microscope (TEM) JEM-2500SE (JEOL, Japan), operated at $200 \mathrm{kV}$, was used to observe the shape and size of the AuNPs. Samples were prepared by adding ethanol into the suspension which was then dropped onto a copper grid before let it dry by exposure to the air for $24 \mathrm{~h}$. Particle size was obtained by measuring diameters of 80 particles viewed in the TEM images. Zeta potential analysis was carried out using a zeta potential analyzer ELS-7300K (Photal Otsuka Electronics, Japan). Data were observed from samples which were left standing for 4 months. The $\mathrm{pH}$ measurement was conducted using pH/lon Meter F-53 (Horiba, Japan).

\section{Results and discussion}

\subsection{Reactive species generated during the AuNP formation}

Reactive species generated during the plasma discharge in alginate aqueous solution containing gold (III) chloride trihydrate were investigated by optical emission spectroscopy (OES). Results are shown in Fig. 2. Appearance of emission peaks corresponding to excited state of atomic hydrogen (i.e., $\mathrm{H}_{\alpha}$ at $\lambda=656 \mathrm{~nm}$; and $\mathrm{H}_{\beta}$ at $\lambda=487 \mathrm{~nm}$ ) and atomic oxygen (i.e., $\mathrm{O} \mathrm{I}$ at $\lambda=$ 778 and $845 \mathrm{~nm}$; and $\mathrm{O} \|$ at $\lambda=743 \mathrm{~nm}$ ) reveals that predominant reactive species occurring in the reaction are $\mathrm{H}$ and $\mathrm{O}$ species. These species come from decomposition of water molecules, the medium used in this study. Emission peaks corresponding to $\mathrm{Na}$ atoms in the alginate polymer are additionally observed (i.e., Na I at $\lambda=590 \mathrm{~nm}$; and $\mathrm{Na} \|$ at $\lambda=618 \mathrm{~nm}$ ). The presence of activated Au species is confirmed by the occurrence of Au II emission peak at $\lambda=$ $401 \mathrm{~nm}$. Study reported by Saito et al. [13] indicates that $\mathrm{H}$ radical functions as a reducing agent of the gold ions for the synthesis of AuNPs by glow discharge in aqueous solution. It should be postulated that, the fast reduction of the gold ions to the AuNPs could be caused by an 
enormous amount of the $\mathrm{H}$ species generated in the reaction.

\subsection{Optical absorption property, shape, and size of the AuNPs}

Optical absorption properties of the AuNPs, caused by a phenomenon known as surface plasmon resonance (SPR), are highly dependent on the shape and size of the nanoparticles. UV-vis absorption spectra of AuNPs-alginate suspensions produced with different discharge times are shown in Fig. 3. Data were collected from samples which were left standing after the discharge for six days. The observed SPR spectra with only one band suggest that the synthesized AuNPs tend to have spherical shape [16]. It is generally known that the plasmon resonance maximum values $\left(\lambda_{\max }\right)$ of the AuNPs shift to the shorter wavelength (blue shift) when the size of the nanoparticles decreases [17]. According to results shown in Fig. 3, sizes of the AuNPs produced without the SPP and with a shorter SPP discharge time (i.e. 5 min) should be larger than that of produced at the longer discharge times. This is confirmed by TEM images shown in Fig. 4, along with average sizes of the nanoparticles shown in Fig. 5. Appearance of the AuNPs produced in water was also investigated as a control test. Clearly, while the AuNPs disperse well in the alginate matrix of all discharge times, aggregation of the particles is observed in water.

Considering the particle shape, the shape of the AuNPs produced in water is non-uniform. Anisotropic shapes of the AuNPs, such as triangular and hexagonal nanoparticles, are also observed in the untreated sample and 5 min discharged sample. However, AuNPs produced at discharge times of $\geq 10$ min tend to be more spherical. For the untreated sample, AuNPs with average size of $46.15 \pm 21.63 \mathrm{~nm}$ are observed. After $5 \mathrm{~min}$ discharge, the average size of the particles is reduced to $23.52 \pm 17.67 \mathrm{~nm}$. Discharge times of $\geq 10 \mathrm{~min}$ lead to a significant decrease in the particle size. The average sizes of the AuNPs are found to be $9.94 \pm$ 
$2.41,8.99 \pm 2.91$, and $7.01 \pm 4.63 \mathrm{~nm}$ for 10,20 , and $30 \mathrm{~min}$ discharged samples, respectively. It should additionally be noted that when the discharge times are $\geq 10 \mathrm{~min}$, we can observe not only the uniformity in shape of the AuNPs but also their narrower size distribution. The reduction in size of the AuNP when the discharge time increased was also observed in the study reported by Saito et al. in which the synthesis was done by the SPP in water with the presence of sodium dodecyl sulfonate functioning as stabilizer [13]. They concluded that the reduction in the particle size was caused by the dissolution of the nanoparticles, owing to the $\mathrm{pH}$ reduction, when the discharge time was prolonged. In their study, the $\mathrm{pH}$ gradually reduced from $\sim 3.15$ to $\sim 2.65$ when the plasma discharge times were 0 and $50 \mathrm{~min}$, respectively. Although the $\mathrm{pH}$ reduction when the discharge time increased is observed in our study as well, $\mathrm{pH}$ values of the AuNPs-alginate suspensions are not as low as in water, i.e., the pH values are in the range of 5.4-5.2 (see Fig. S1). Therefore, in case of discharge in the alginate matrix we additionally propose that the reduction in size of the particles when the discharge times were increased should relate to the concept of nucleation and growth of the crystal. Briefly, after the plasma is generated in the reactor, the AuNPs initially form and become "nucleation site". After a nucleation site has created it must grow. The nucleation sites are necessary for the growth of the crystal, but excess nucleation sites will cause smaller crystal size. When the reactive species are generated at an adequate discharge time, large amount of nucleation sites are produced. This is the reason why smaller sizes of the AuNPs are observed at the longer discharge times. Furthermore, the sizes of the AuNPs synthesized by Saito et al. are significantly larger than that of synthesized in this study, i.e., sizes of AuNPs were found to be 150,50 , and $20 \mathrm{~nm}$ when the discharge times were 1 , 5, and 45 min, respectively [13]. The observed smaller in size of the AuNPs produced in the alginate solution could be caused by the same phenomenon found in our previously reported work [15] that the alginate polymer can form cavities and function as a template for the growth of 
the gold clusters. As a consequence, the growth of the particles is limited in the alginate matrix.

Considering the production of AuNPs in the same matrix, the average size of the AuNPs stabilized in alginate solution produced in this study is larger than that of produced by the SPS process [15]. Specifically, the average particle diameters of AuNPs produced by the SPS process was found to be $3.54 \pm 1.04 \mathrm{~nm}$ when the particles were produced in the same matrix of equivalent concentration and discharge time (0.5\%w/v alginate, $10 \mathrm{~min}$ discharge). While this remarkable shape difference is observed, the actual explanation of possible phenomenon is not certain at the present time.

\subsection{Role of the alginate matrix}

Although the reduction of gold ions to AuNPs by the SPP is principally due to the occurrence of $\mathrm{H}$ radicals from plasma generation, ability of alginate to function as a reducing agent for AuNP formation has also previously been reported [18]. Fig. 6 shows appearance of the AuNPs-alginate suspensions produced with different discharge times. Pictures were taken immediately after the discharge and after samples were left standing for one and six days. It is clearly seen from Fig. 6 that while the as-prepared AuNPs-alginate suspensions produced at discharge times of 10,20, and 30 min exhibit ruby-red color, the untreated suspension and the suspension produced at a discharge time of $5 \mathrm{~min}$ exhibit no color and light pink-purple, respectively. When the left standing time was prolonged, color intensity of the suspensions; especially the untreated sample and the sample produced with a discharge time of $5 \mathrm{~min}$, significantly increases, leading to the suspensions of purple and dark pink-purple color, respectively. In order to indicate the magnitude of the color increment, intensity change of the UV-vis spectra was additionally carried out. Results are shown in Fig. 7. It is clearly seen that the increase in the color intensity is observed only for the untreated and the 5 min treated 
suspensions, indicating that $0.2 \mathrm{mM} \mathrm{HAuCl}_{4} \cdot 3 \mathrm{H}_{2} \mathrm{O}$ in $90 \mathrm{~mL}$ of $0.5 \% \mathrm{w} / \mathrm{v}$ alginate solution can be reduced completely to the AuNPs within $10 \mathrm{~min}$. Furthemore, the increment of the color intensity is more remarkable for 5 min discharged sample compared with that of the untreated sample. This finding suggests that after the gold precursor is reacted with the plasma, reactive species generated during the SPP still active and continuously react with some unreacted part of the gold precursor. Concurrently, reduction reaction is lengthened for a period of time.

Role of the alginate matrix in the stabilization of AuNP suspension was confimed by comparing the stability of the AuNP suspension produced in alginate solution and water (30 min discharge). Results are shown in Fig. 8. It is found that while the AuNPs discharged in water form aggregates and precipitate since 2 days of the observation, the AuNPs discharged in the alginate solution still disperse well (Fig. 8a). The shift and change of the UV-vis patterns of the AuNPs suspensions produced in water measured immediately after the discharge and after samples were left standing are also investigated and shown in Fig. S2. Quantitative analysis (zeta potential measurement, Fig. 8b) reveals that the AuNPs-alginate suspensions produced within all discharge times exhibit good stability until the suspensions were left standing for 4 months. Specifically, all AuNPs-alginate suspensions have zeta potential values more negative than -30 $\mathrm{mV}$ which is the general dividing line to determine whether the suspension is stable or not [15]. These results suggest that sodium alginate solution acts both as a stabilizer and a reducing agent for the AuNPs.

\section{Conclusions}

We report an influence of the discharge time of the SPP on the formation of AuNPs in the alginate aqueous solution. A size reduction of the AuNPs is observed when the discharge time is increased up to $10 \mathrm{~min}$. After that the average particle sizes tend to be unchanged. 
Furthermore, discharge times of $\geq 10$ min lead to the formation of particles with more spherical shape and uniform size. Comparing with the AuNPs produced by the SPS process, the average size of the AuNPs stabilized in alginate solution produced in this study is larger. Although the observations reveal that alginate itself can act both as a reducing agent and a stabilizer for the single-step synthesis of the AuNPs, the application of the SPP offers the advantages of fast completion, controllability of shape and size, and resulting in a ready-to-use product.

\section{Acknowledgements}

We greatly appreciate the financial support from the Core Research for Evolutional Science and Technology (CREST) of Japan Science and Technology (JST) Agency.

\section{References}

[1] Saha K, Agasti SS, Kim C, Li X, Rotello VM. Gold nanoparticles in chemical and biological sensing. Chem Rev2012;112:2739-2779.

[2] Dreaden EC, Austin LA, Mackey MA, El-Sayed MA. Size matters: gold nanoparticles in targeted cancer drug delivery. Ther Deliv 2012;3:457-478.

[3] Park C, Youn H, Kim H, Noh T, Kook YH, Oh ET, Park HJ, Kim C. Cyclodextrin-covered gold nanoparticles for targeted delivery of an anti-cancer drug. J Mater Chem 2009;19:23102315.

[4] Sonavane G, Tomoda K, Makino K. Biodistribution of colloidal gold nanoparticles after intraveneous administration: effect of particle size. Colloids Surf B 2008;66:274-280.

[5] Kim JH(a), Kim JH(b), Kim KW, Kim MH, Yu YS. Intravenously administered gold nanoparticles pass through the blood-retinal barrier depending on the particle size, and induce no retinal toxicity. Nanotechnology 2009;20:505101. 
[6] Majzik A, Fülöp L, Csapó E, Bogár F, Martinek T, Penke B, Bíró G, Dékány I.

Functionalization of gold nanoparticles with amino acid, $\beta$-amyloid peptides and fragment. Colloids Surf B 2010;81:235-241.

[7] Singh DK, Jagannathan R, Khandelwal P, Abraham PM, Poddar P. In situ synthesis and surface functionalization of gold nanoparticles with curcumin and their antioxidant properties: an experimental and density functional theory investigation. Nanoscale 2013;5:1882-1893.

[8] Venkatpurwar V, Shiras A, Pokharkar V. Porphyran capped gold nanoparticles as a novel carrier for delivery of anticancer drug: in vivo cytotoxicity study. Int J Pharm 2011;409:314 320.

[9] Irure A, Marradi M, Arnáiz B, Genicio N, Padroc D, Penadés S. Sugar/gadolinium-loaded gold nanoparticles for labelling and imaging cells by magnetic resonance imaging. Biomater Sci 2013;1:658-668.

[10] Lim SY, Lee JS, Park CB. In situ growth of gold nanoparticles by enzymatic glucose oxidation within alginate gel matrix. Biotechnol Bioeng 2010;105:210-214.

[11] Kodiyan A, Silva EA, Kim J, Aizenberg M, Mooney DJ. Surface modification with alginate-derived polymers for stable, protein-repellent, long-circulating gold nanoparticles. ACS Nano 2012;6:4796-4805.

[12] Takai O. Solution plasma processing (SPP). Pure Appl Chem 2008;80:2003-2011.

[13] Saito N, Hieda J, Takai O. Synthesis process of gold nanoparticles in solution plasma. Thin Solid Films 2009;518:912-917.

[14] Hu XL, Takai O, Saito N. Synthesis of gold nanoparticles by solution plasma sputtering in various solvents. J Phys: Conf Ser 2013;417:012030.

[15] WatthanaphanitA, Panomsuwan G, Saito N. A novel one-step synthesis of gold 
nanoparticles in an alginate gel matrix by solution plasma sputtering. RSCAdv 2014;4:1622-1629.

[16] El-Brolossy TA, Abdallah T, Mohamed MB, Abdallah S, Easawi K, Negm S, Talaat H. Shape and size dependence of the surface plasmon resonance of gold nanoparticles studied by Photoacoustic technique. Eur Phys J Spec Top 2008;153:361-364.

[17] Pal A, Esumi K, Pal T. Preparation of nanosized gold particles in a biopolymer using UV photoactivation. J Colloid Interface Sci 2005;288:396-401.

[18] Pal A, Esumi K. Photochemical synthesis of biopolymer coated $\mathrm{Au}_{\text {core }}-\mathrm{Ag}_{\text {shell }}$ type bimetallic nanoparticles. J Nanosci Nanotechnol 2007;7:2110-2115. 


\section{Figure captions}

Fig. 1 Schematic illustration of the experimental setup.

Fig. 2 OES spectra of the plasma generated in alginate aqueous solution containing gold (III) chloride trihydrate.

Fig. 3 UV-vis absorption spectra of AuNPs-alginate suspensions produced with different discharge times. Data were collected from samples which were left standing after the discharge for six days.

Fig. 4 TEM images of the AuNPs produced in (a) water (30 min discharge), and (b-f) alginate aqueous solution with different discharge times.

Fig. 5 Average sizes of the AuNPs produced in alginate aqueous solution with different discharge times.

Fig. 6 Appearance of the AuNPs-alginate suspensions produced with different discharge times. Pictures were taken immediately after the discharge (As-prepared suspensions) and after samples were left standing for one and six days.

Fig. 7 Change in UV-vis absorption spectra intensity of the AuNPs-alginate suspensions produced with different discharge times. Data were collected immediately after the discharge and after samples were left standing for several time intervals.

Fig. 8 (a) AuNP suspensions produced in water and alginate aqueous solution (30 min discharge), before and after allowed to be settled. (b) Zeta potential values of the AuNPs-alginate suspensions produced with different discharge times. Data were collected after the suspensions were left standing for 4 months. 
Figure 1.
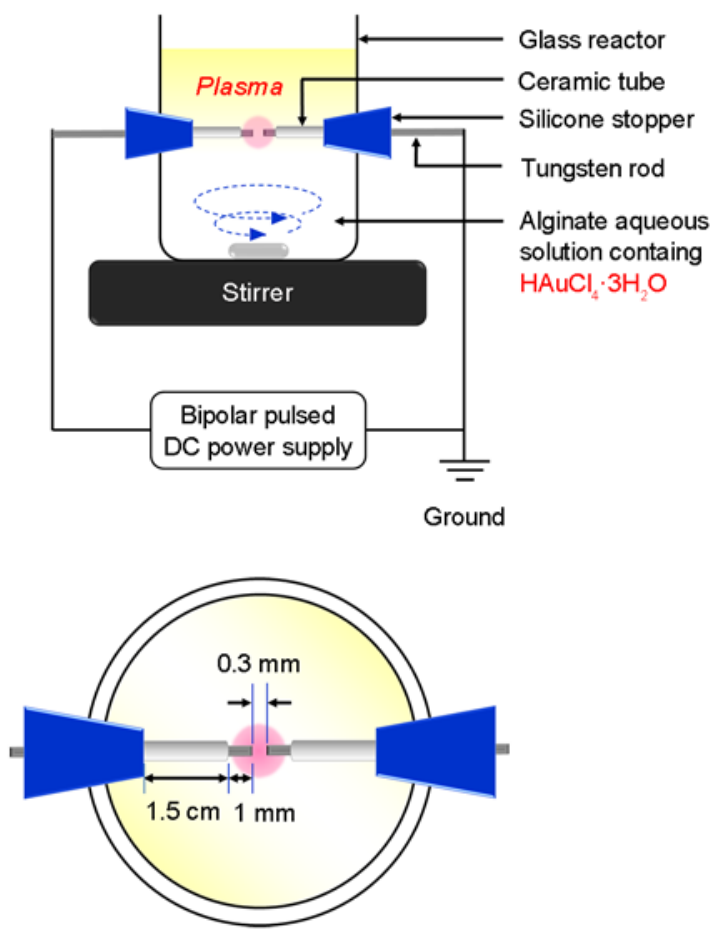
Figure 2.

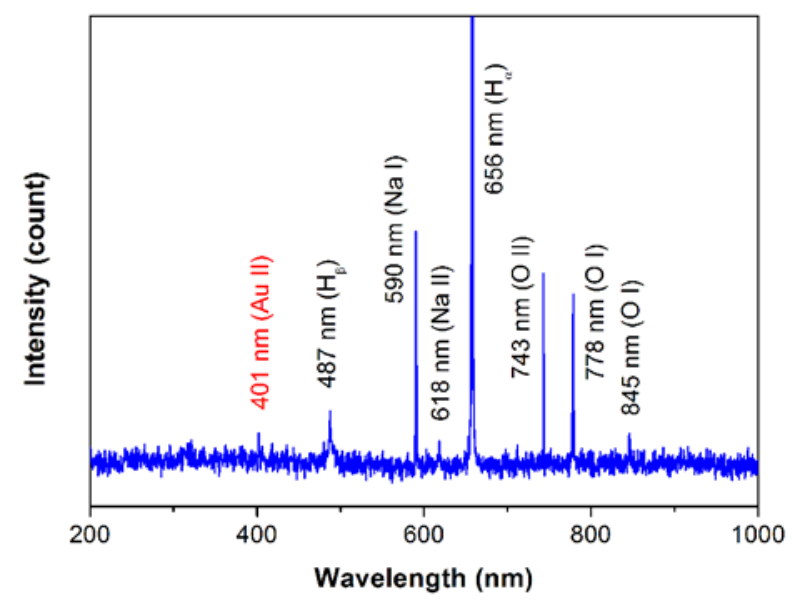


Figure 3.

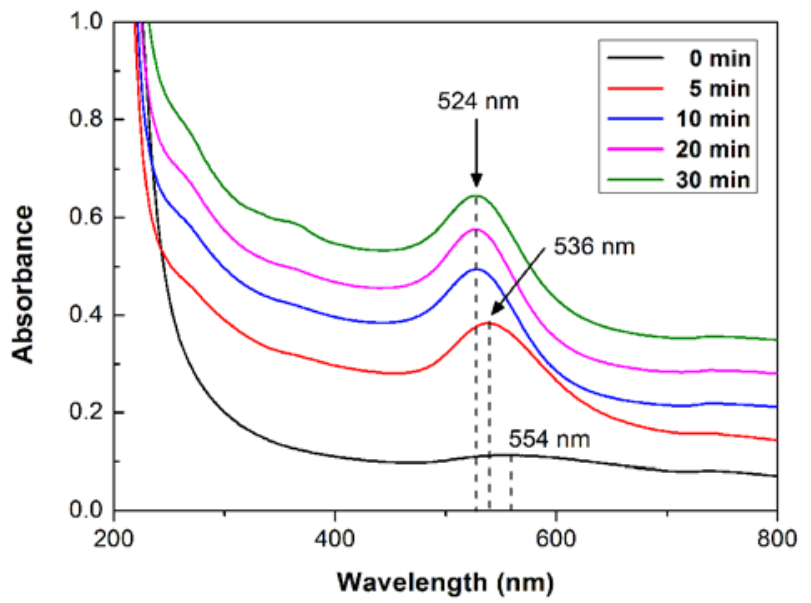


Figure 4 .
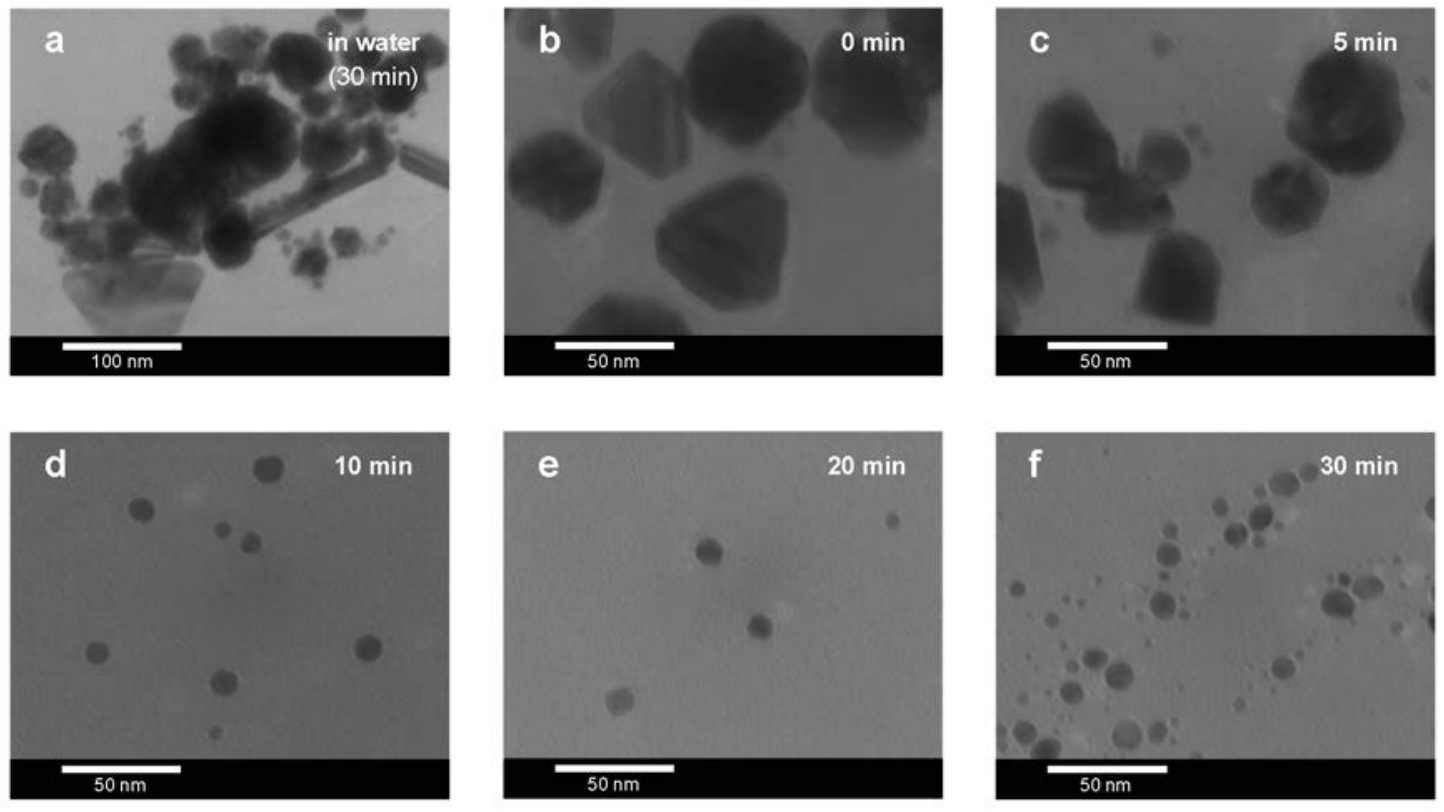
Figure 5.

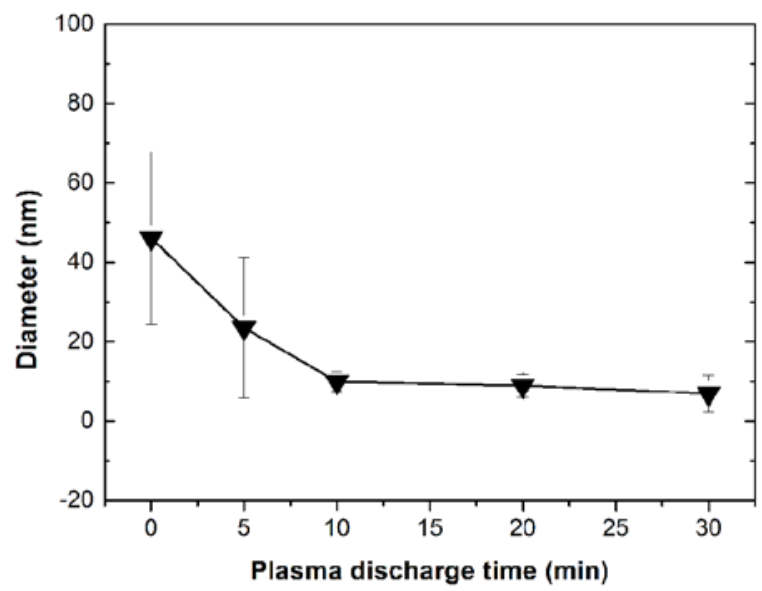


Figure 6.

As-prepared suspensions

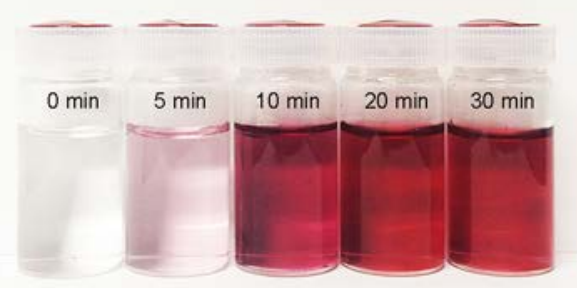

After 1 day

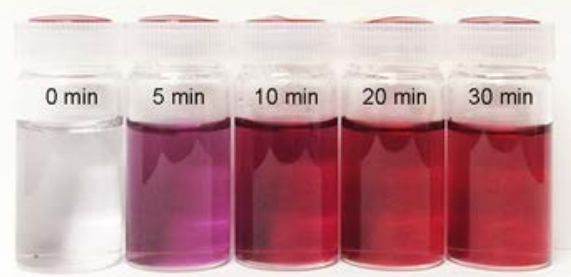

After 6 days

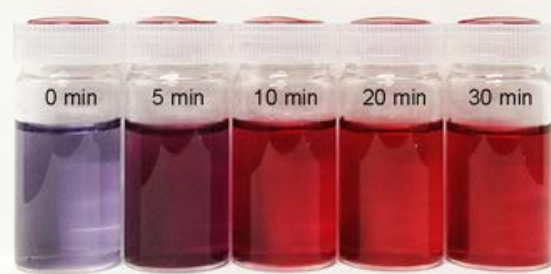


Figure 7.

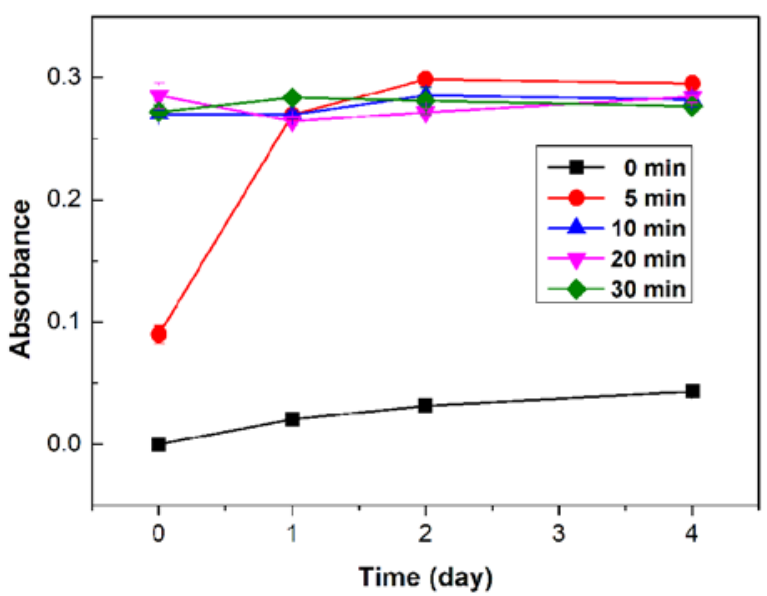


Figure 8.

a

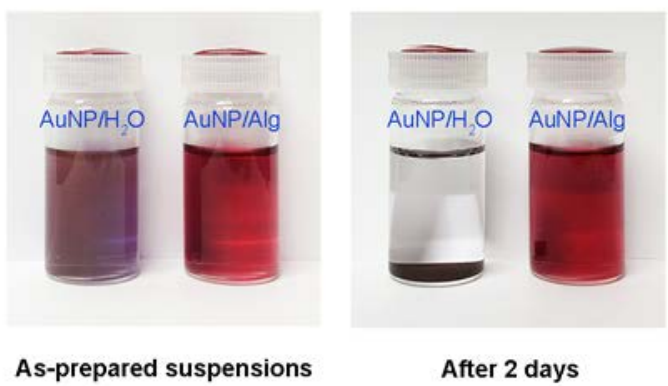

b

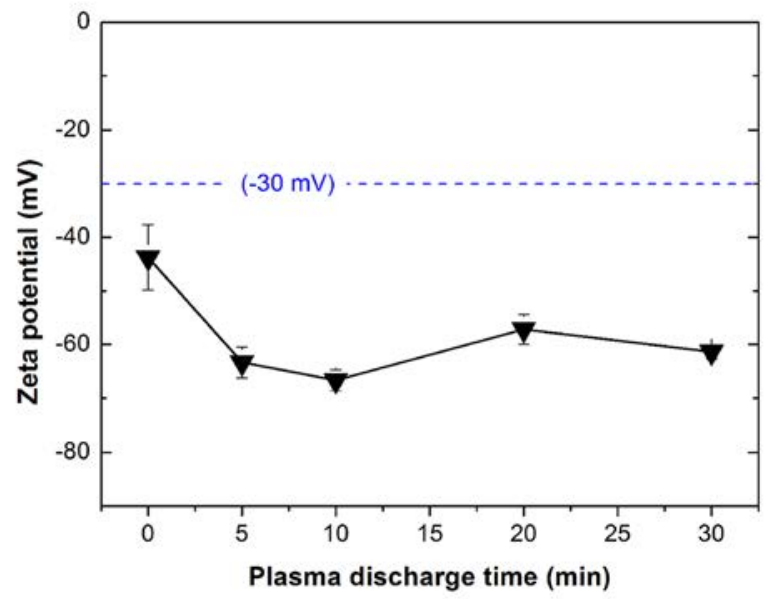

